\title{
The 15 item Geriatric Depression Scale had perfect sensitivity but low specificity for detecting depression in elderly people
}

Arthur A, Jagger C, Lindesay J, et al. Using an annual over-75 health check to screen for depression: validation of the short Geriatric Depression Scale (GDS15) within general practice. Int J Geriatr Psychiatry 1999 Jun;14:431-9.

QUESTION: In elderly people ( $\geqslant 75$ y of age), is the shorter, 15 item Geriatric Depression Scale (GDS15) a useful screening tool for detecting depression and anxiety?

\section{Design}

Blinded comparison of GDS15 with diagnoses based on the $I C D-10$ criteria for mood disorders.

\section{Setting}

A large general practice in Melton Mowbray, Leicestershire, UK.

\section{Patients}

201 patients (median age 79 y, 57\% women) who were $\geqslant 75$ years of age, were not in residential care, and had completed the diagnostic interview after completing the GDS15 during a health check. All patients who scored $\geqslant 5$ on the GDS15, 1 in 2 patients who scored 3 or 4 , and 1 in 4 of remaining patients were asked to complete the diagnostic interview $(n=257)$.

Description of test and diagnostic standard The GDS15 was given by a practice nurse during regular annual home health checks. A geriatric psychiatrist and a research nurse, who were blind to the GDS15 results, used the Schedules for Clinical Assessment in Neuropsychiatry to make $I C D$ - 10 diagnoses.

\section{Main outcome measures}

Sensitivity, specificity, and likelihood ratios.

\section{Main results}

Results are shown in the table.

\section{Conclusions}

In elderly people ( $\geqslant 75 \mathrm{y}$ of age), the shorter, 15 item Geriatric Depression Scale (GDS15) had perfect sensitivity but low specificity for detecting depression when a cutoff score of 3 was used. The sensitivity and specificity of the GDS15 for detecting anxiety disorders were not high.

Test characteristics of GDS15 for detecting depression and anxiety*

\begin{tabular}{llllll} 
Outcomes & $\begin{array}{l}\text { Cutoff } \\
\text { score }\end{array}$ & Sensitivity (95\% CI) & Specificity (CI) & +LR & -LR \\
Depression & $2 / 3$ & $100 \%(88$ to 100$)$ & $72 \%(68$ to 76$)$ & 3.6 & 0.0 \\
\cline { 2 - 6 } & $3 / 4$ & $80 \%(61$ to 92$)$ & $82 \%(78$ to 85$)$ & 4.3 & 0.2 \\
\hline Anxiety & $2 / 3$ & $79 \%(54$ to 94$)$ & $69 \%(65$ to 73$)$ & 2.6 & 0.3 \\
\cline { 2 - 6 } & $3 / 4$ & $68 \%(43$ to 87$)$ & $80 \%(76$ to 83$)$ & 3.4 & 0.4
\end{tabular}

*GDS15=15 item Geriatric Depression Scale. +LR=likelihood ratio for a positive test result; $-\mathrm{LR}=$ likelihood ratio for a negative test result. Diagnostic terms defined in glossary; LRs and Cl calculated from data in article. Values are weighted by the inverse of the sampling fraction from the first (administration of GDS15) to second (diagnostic interview) stage.

\section{COMMENTARY}

There is no doubt that most depression in older people goes undetected and untreated. ${ }^{1}$ Patients and doctors seem to collude to avoid the stigma and resignation that the diagnosis brings. This happens even though a simple intervention (a pill) has been shown to be helpful for over one half of this group, and a range of talking therapies seem to be equally effective. ${ }^{2}$ One way of improving the detection of depression is by using screening questionnaires. Checklists are generally disliked by healthcare professionals and may be tiresome for older clients. Hence the interest in looking at the efficacy of brief scales (even though the briefest scale possible [BSP] "Are you feeling depressed?" has yet to be implemented universally). In fact, the 15 questions in the GDS15 are similar to the 8 questions in the EBAS DEP, raising the possibility of one day unifying them (although the ensuing acronym would be unbearably long). The main difference between the studies is the populations they examine. The GDS15 was studied in people $\geqslant 75$ years of age who lived in the community as part of their legislated (in the UK) annual health checkups. The EBAS DEP was used in a smaller group of people who were $\geqslant 65$ years of age in Germany and lived in an old people's home or hospital or attended a clinic. Each study looked at how different cutoff scores for the scales compared with a gold standard of clinical criteria for depression. There was the usual tradeoff between sensitivity and specificity. If only 3 questions need to be answered "yes" to screen the patient as positive, then both scales are very sensitive (picking up all the small number of depressed patients in each study). The scales also identify a lot of "false-positives", however, which is inevitable when the continued on next page

Source of funding: Nuffield Provincial Hospitals Trust.

For correspondence: $\mathrm{Mr}$ A Arthur, Department of Epidemiology and Public Health, University of Leicester, 22-28 Princess Road West, Leicester

LE1 6TP, UK. Fax +44 (0)166 4481427 Emailaja@le.ac.uk 


\section{The Even Briefer Assessment Scale for Depression in German was accurate for identifying depression in elderly patients}

Weyerer S, Killmann U, Ames D, et al. The Even Briefer Assessment Scale for Depression (EBAS DEP): its suitability for the elderly in geriatric care in English- and German-speaking countries. Int J Geriatr Psychiatry 1999 Jun;14:473-80.

QUESTION: In elderly people, is the German version of the Even Briefer Assessment Scale for Depression (EBAS DEP) accurate for identifying depression?

Design

A blinded comparison of the EBAS DEP with clinical diagnoses based on the Feighner criteria.

\section{Setting}

An old age home, a day clinic, and a psychiatric hospital in Mannheim, Germany.

\section{Participants}

71 participants who were $\geqslant 65$ years of age.

Description of test and diagnostic standard The EBAS DEP has 8 questions on depressive mood, worries, suicidal thoughts, and vegetative symptoms, and scores range from 0 (not depressed) to 8 (very depressed). The diagnostic standard was diagnoses made by a psychiatrist or psychiatric nurse who used the Feighner criteria and who were blind to EBAS DEP scores.

Main outcome measures

Sensitivity, specificity, and likelihood ratios.

\section{Main results}

The table shows the results. The best cutoff point was $4 / 5$ with a sensitivity of $93 \%$ and a specificity of $85 \%$. When receiver operating characteristic (ROC) curves were constructed, the area under the EBAS DEP ROC curve was similar to that of the full 21 item version of the Brief Assessment Scale for Depression.

\section{Conclusion}

The German version of the Even Briefer Assessment Scale for Depression had high sensitivity and specificity for detecting depression in elderly patients.

Test characteristics of the German version of the Even Briefer Assessment Scale for Depression*

\begin{tabular}{lllll} 
Cutoff score & Sensitivity & Specificity & +LR & -LR \\
\hline $2 / 3$ & $100 \%$ & $47 \%$ & 1.9 & 0.0 \\
\hline $3 / 4$ & $100 \%$ & $68 \%$ & 3.1 & 0.0 \\
\hline $4 / 5$ & $93 \%$ & $85 \%$ & 6.2 & 0.8 \\
\hline
\end{tabular}

*+LR=likelihood ratio for a positive test result; $-\mathrm{LR}=$ likelihood ratio for a negative test result. Diagnostic terms defined in glossary; LRs calculated from data in article.
Source of funding: German Ministry of Research and Technology.

For correspondence: Dr $S$ Weyerer, Psychogeriatric Research Unit, Central Institute of Mental Health, J5, D-68159 Mannheim, Germany. Fax +4962123429

\section{COMMENTARY—continued from previous page}

prevalence of a disorder is low. This may mean further unnecessary clinical interviews. No one knows whether those with false positive results may also benefit from further interventions. The take home message is that both scales are useful for screening elderly people. Every silver lining has a cloud, however. Once detected, depressed older people will need to be treated properly. Even with widely practicable treatments such as drugs, evidence exists that they are not always used well and that if quality is improved, so are outcomes. ${ }^{3}$

If the average geriatric psychiatrist covers the area of 50 general practitioners (GPs), Arthur $e$ al s figures suggest that the GDS15 would screen 2100 people $\geqslant 75$ years of age as positive, of whom 400 would be depressed. This would generate too much work for existing services. Perhaps this is one reason why GPs are not presently using depression scales in their annual screening of people who are $\geqslant 75$ years of age. ${ }^{4}$

Rob Butler, MBBS, MRCPsych Edgeware Community Hospital Brain Injury Unit Middlesex, $U K$

1 Katona C, Freeling P, Hinchcliffe K, et al. Recognition and management of depression in late life in general practice: consensus statement. Primary Care Psychiatry 1995;1:107-13.

2 Depression Guideline Panel. Depression in primary care. Rockville, MD: Agency for Health Care Policy and Research, 1993.

3 Rutz W, von Knorring L, Walinder J. Long-term effects of an educational program for general practitioners given by the Swedish Committee for the Prevention and Treatment of Depression. Acta Psychiatr Scand 1992;85:83-8.

4 Wilkieson CA, Campbell A, McWhirter MF, et al. Standardization of health assessments for patients aged 75 years and over: 3 years experience in the Forth Health Board area. Br J Gen Pract 1996;46:307-8. 